\title{
OÚRICURI
}

\section{OCORRÊNCIA DE FUNGOS DEMÁCEOS EM FARPAS DE PORTÕES DE MADEIRA, NO MUNICÍPIO DE SOURE, PARÁ}

\author{
Adriane Nunes FARIAS ${ }^{1 *}$; Adriano BIANCALANA ${ }^{2}$; Fernanda Simas Corrêa BIANCALANA ${ }^{2}$
}

\author{
${ }^{1}$ Graduada em Ciências Biológicas, Universidade Federal do Pará. Laboratório de Microbiologia e \\ Parasitologia, Rua Décima Terceira, Umirizal, CEP: 68870-000, Soure - PA, Brasil. *Autor correspondente - \\ E-mail: adrianenunes5@gmail.com \\ ${ }^{2}$ Faculdade de Ciências Biológicas, Universidade Federal do Pará - Campus Universitário do Marajó/Soure, \\ CEP: 68870-000, Soure - PA, Brasil. E-mail: biancalana@ufpa.br; fbiancalana@ufpa.br. \\ Recebido: 02.12.2019 Aceito: 25.05.2020 \\ http://doi.org/10.29327/ouricuri.9.2-2
}

\begin{abstract}
Resumo: O presente trabalho objetivou verificar a ocorrência de fungos demáceos em farpas de portões de madeira no município de Soure, Pará. As amostras de farpas foram coletadas na estação seca de 2018, onde se obteve 40 amostras, e nas estações seca e chuvosa de 2019, obtendo-se 80 amostras. A cada estação, amostras de farpas de vinte portões da zona urbana e de vinte da zona rural foram coletadas, totalizando 120 amostras advindas de 40 portões. Para a coleta das farpas dos portões utilizou-se pinça, papel alumínio e sacos plásticos. As amostras foram levadas para o Laboratório de Microbiologia e Parasitologia, da Universidade Federal do Pará (UFPA), campus do Marajó - Soure, semeadas em placas de Petri, contendo meio de cultura Batata Dextrose Ágar (BDA), para a obtenção dos isolados fúngicos, e incubadas por um período de 5 a 7 dias, em temperatura ambiente. Após o crescimento dos isolados fúngicos, lâminas foram preparadas e analisadas em microscopia de luz para identificação de seus gêneros. Foi constatado o crescimento de 137 colônias de fungos filamentosos, sendo 55 dessas pertencentes aos fungos demáceos dos gêneros: Cladosporium sp., Curvularia sp., Exophiala sp., Fonsecaea sp. e Phialophora sp., o que corresponde a 46 de 111 amostras de farpas analisadas. As 55 colônias de fungos demáceos encontradas estão distribuídas entre as estações seca de 2018, sendo 11 colônias observadas em portões da zona urbana e 15 da zona rural, seca de 2019, com 4 colônias na área urbana e 12 na área na rural, e chuvosa de 2019, com o crescimento de 8 colônias na zona urbana e de 5 na zona rural. Phialophora sp. foi o gênero de fungos demáceos mais abundante. Há fungos demáceos colonizando farpas de madeira de portões, o que é um risco de contaminação para população que os manuseia diariamente.
\end{abstract}

Palavras-chave: Amazônia; Fungos Melanizados; Cromoblastomicose; Farpas.

\section{OCCURRENCE OF DEMACEOUS FUNGI IN WOODEN GATE BARS, IN THE MUNICIPALITY OF SOURE, PARÁ}

\begin{abstract}
The present work aimed to verify the occurrence of demaceous fungi in barbs of wooden gates in the municipality of Soure, Pará state. The barbs samples were collected in the dry season of 2018, where 40 samples were obtained, and in the dry and rainy seasons of 2019, obtaining 80 samples. At each station, samples of barbs from twenty gates in the urban area and twenty in the rural area were collected, totaling 120 samples from 40 gates. To collect the barbs from the gates, tweezers, aluminum foil and plastic bags were used. The samples were taken to the Microbiology and Parasitology Laboratory, Federal University of Pará (UFPA), Marajó - Soure campus, sown in Petri dishes, containing Potato Dextrose Agar (BDA) culture medium, to obtain the fungal isolates, and incubated for a period of 5 to 7 days, at room temperature. After the growth of fungal isolates, slides were prepared and analyzed under light microscopy to identify their genera. It was observed
\end{abstract}

Revista Ouricuri, Juazeiro, Bahia, v.9, n.2. p.011-024. jul./dez., 2019.

http://www.revistas.uneb.br/index.php/ouricuri | ISSN 2317-0131 
the growth of 137 colonies of filamentous fungi, 55 of which belong to the demaceous fungi of the genera: Cladosporium sp., Curvularia sp., Exophiala sp., Fonsecaea sp. and Phialophora sp., which corresponds to 46 out of 111 barbs samples analyzed. The 55 colonies of demaceous fungi found are distributed between the dry seasons of 2018, with 11 colonies observed in urban gates and 15 in the rural zone, drought in 2019, with 4 colonies in the urban area and 12 in the rural area, and rainy 2019 , with the growth of 8 colonies in the urban area and 5 in the rural area. Phialophora $s p$. was the most abundant demaceous fungus genus. There are demaceous fungi colonizing wooden barbs from gates, which is a risk of contamination for the population that handles them daily.

Keywords: Amazon; Melanized Fungi; Chromoblastomycosis; Barbs.

\section{OCURRENCIA DE HONGOS DEMACEOS EN ASTILLAS DE PUERTA DE MADERA, EN EL MUNICIPIO DE SOURE, PARÁ}

Resumen: El presente trabajo tuvo como objetivo verificar la presencia de hongos demaceos en astillas de puertas de madera en el municipio de Soure, Pará. Las muestras de astillas se recolectaron en la estación seca de 2018, donde se obtuvieron 40 muestras, y en las estaciones seca y lluviosa de 2019, obteniendo 80 muestras. En cada estación, se recolectaron muestras de astillas de veinte puertas en el área urbana y veinte en el área rural, totalizando 120 muestras de 40 puertas. Para recoger las astillas de las puertas, se utilizaron pinzas, papel de aluminio y bolsas de plástico. Las muestras fueron llevadas al Laboratorio de Microbiología y Parasitología, Universidad Federal de Pará (UFPA), campus Marajó - Soure, sembradas en placas Petri, que contienen medio de cultivo de agar papa dextrosa (BDA), para obtener los hongos aislados, y se incubaron durante un período de 5 a 7 días, a temperatura ambiente. Después del crecimiento de aislados fúngicos, se prepararon portaobjetos y se analizaron con microscopía óptica para identificar sus géneros. Se observó el crecimiento de 137 colonias de hongos filamentosos, 55 de los cuales pertenecen a los hongos demaceos de los géneros: Cladosporium sp., Curvularia sp., Exophiala sp., Fonsecaea sp. y Phialophora sp., que corresponde a 46 de 111 muestras de astillas. Las 55 colonias de hongos demaceos encontrados se distribuyen entre las estaciones secas de 2018, con 11 colonias observadas en puertas urbanas y 15 en la zona rural, sequía en 2019, con 4 colonias en el área urbana y 12 en el área rural, y lluviosas 2019, con el crecimiento de 8 colonias en el área urbana y 5 en el área rural. Phialophora $s p$. fue el género más abundante del hongos demaceos. Hay hongos demaceos que colonizan astillas de madera de las puertas, lo que representa un riesgo de contaminación para la población que los maneja a diario.

Palabras clave: Amazon; Hongos Melanizados; Cromoblastomicosis; Astillas.

\section{INTRODUÇÃO}

Os fungos demáceos ou melanizados constituem um grupo caracterizado por apresentar melanina em sua parede celular. Esta característica, confere uma coloração mais escura a suas hifas e conídios, além de torná-los mais resistente a condições extremas, como radiação ultravioleta (UV) e altas temperaturas (Badali et al., 2008; Najafzadeh et al., 2011; Zolnerkevic, 2011). São organismos encontrados especialmente na água, solo, plantas e matéria orgânica em decomposição, com distribuição por todo o mundo (Fan et al., 2008).

Há pouco tempo, tem sido apresentada a expressão "melanizados" como a mais correta ao invés de demáceos ou pretos. Considera-se que a melanina que se localiza na parede celular seja um fator de virulência (Revankar, 2006;2010). O pigmento possui muitos papeis que podem explicar a patogenicidade de alguns fungos demáceos, entre eles a capacidade de proteger esses indivíduos 
de radicais livres gerados imunologicamente, moléculas essas que provocam a morte de vários microrganismos (Jacobson, 2000).

Segundo Shelton et al. (2002), a contaminação por esses fungos patogênicos ocorre por meio de pequenos traumas ou aspiração, acontecimentos quase imperceptíveis no cotidiano. Alguns autores relatam que a entrada desses microrganismos no organismo humano, normalmente, acontece quando um elemento orgânico infectado com conídios penetra o tecido do indivíduo (Cucé et al., 1983; Castro 1992; Rubin et al., 1992).

Dentro do grupo de fungos demáceos, há mais de 70 gêneros associados a doenças, entre eles estão: Fonsecaea, Exophiala, Cladophialophora, Phialophora e Rhinocladiella (Vicente et al., 2001; Afsarian et al., 2012). Indivíduos do gênero Exophiala têm sido identificados como alguns dos causadores de doenças como cromoblastomicose, feohifomicose e micetoma, algumas delas originadas por inoculação traumática (Gold et al., 1994; Fothergill, 1996).

De acordo com sua aparência no tecido, as micoses subcutâneas causadas por fungos demáceos são classificadas como: cromoblastomicose com corpos fumagoides, feo-hifomicose com presença de hifas septadas demáceas, e eumicetoma com grãos constituídos também de hifas septadas demáceas (Hoffmann, 2011; Wong e Revankar, 2016). Estas doenças não se mostram de modo invasivo ou disseminado, entretanto, geram um impacto relevante a saúde pública, já que não são de fácil controle e manifestam reaparecimentos, precisando de eficácia no diagnóstico para assim haver um tratamento produtivo (Queiroz-Telles et al., 2011).

No Brasil, a Amazônia é tida como a principal região de ocorrência de cromoblastomicose devido apresentar condições favoráveis como: temperatura, precipitação e umidade (Nimer, 1989). A região em destaque apresenta clima tropical, quente e úmido, e alta variedade de matéria orgânica com bastante substratos, o que pode favorecer o crescimento fúngico (Reis, 1982).

O principal ponto de incidência da cromoblastomicose na Amazônia é o Estado do Pará. Ele possui a segunda maior área da região, cerca de 1.253.164,5 km², além de ser cruzado pelo Rio Amazonas que termina, próximo ao Arquipélago de Marajó, no Oceano Atlântico (Santos, 1993). De acordo com Silva et al. (1999), nos últimos 55 anos, foram registrados 325 casos de cromoblastomicose no estado.

Mesmo com toda relevância ambiental, médica e biotecnológica, dada a este grupo fúngico, se conhece pouco sobre a sua biodiversidade e seus ambientes de ocorrência natural (Zhao et al., 2010).

Tendo em vista que os fungos demáceos apresentam espécies patogênicas responsáveis por causar micoses subcutâneas severas em seres humanos e que portões de madeira são muito utilizados no município, este trabalho teve como objetivo verificar a ocorrência de fungos demáceos em farpas de portões de madeira no município de Soure, Pará. 


\section{METODOLOGIA}

O estudo foi realizado no município de Soure, Pará, o qual está localizado na região litorânea da Ilha de Marajó. Foram avaliados portões residenciais das zonas urbana e rural, incluindo algumas porteiras de fazenda do município.

As farpas foram coletadas na estação seca de 2018, onde se obteve 40 amostras, e nas estações seca e chuvosa de 2019, obtendo-se 80 amostras. A cada estação, vinte amostras de farpas da zona rural e vinte da zona urbana foram coletadas, totalizando 120 amostras advindas de 40 portões, vinte de cada zona. Alguns portões foram substituídos por portões de ferro durante a pesquisa, o que levou a exclusão dos mesmos e de suas amostras, resultando em 37 portões e 111 amostras.

Portões de diferentes bairros foram selecionados através da amostragem aleatória simples por sorteio (Lopes, 2003). Somente os portões que apresentavam farpas, e que eram manuseados diariamente pelos moradores (Figura 1), foram escolhidos para a amostragem. Todas as coletas, nas diferentes estações e anos, foram amostrados nos mesmos portões.
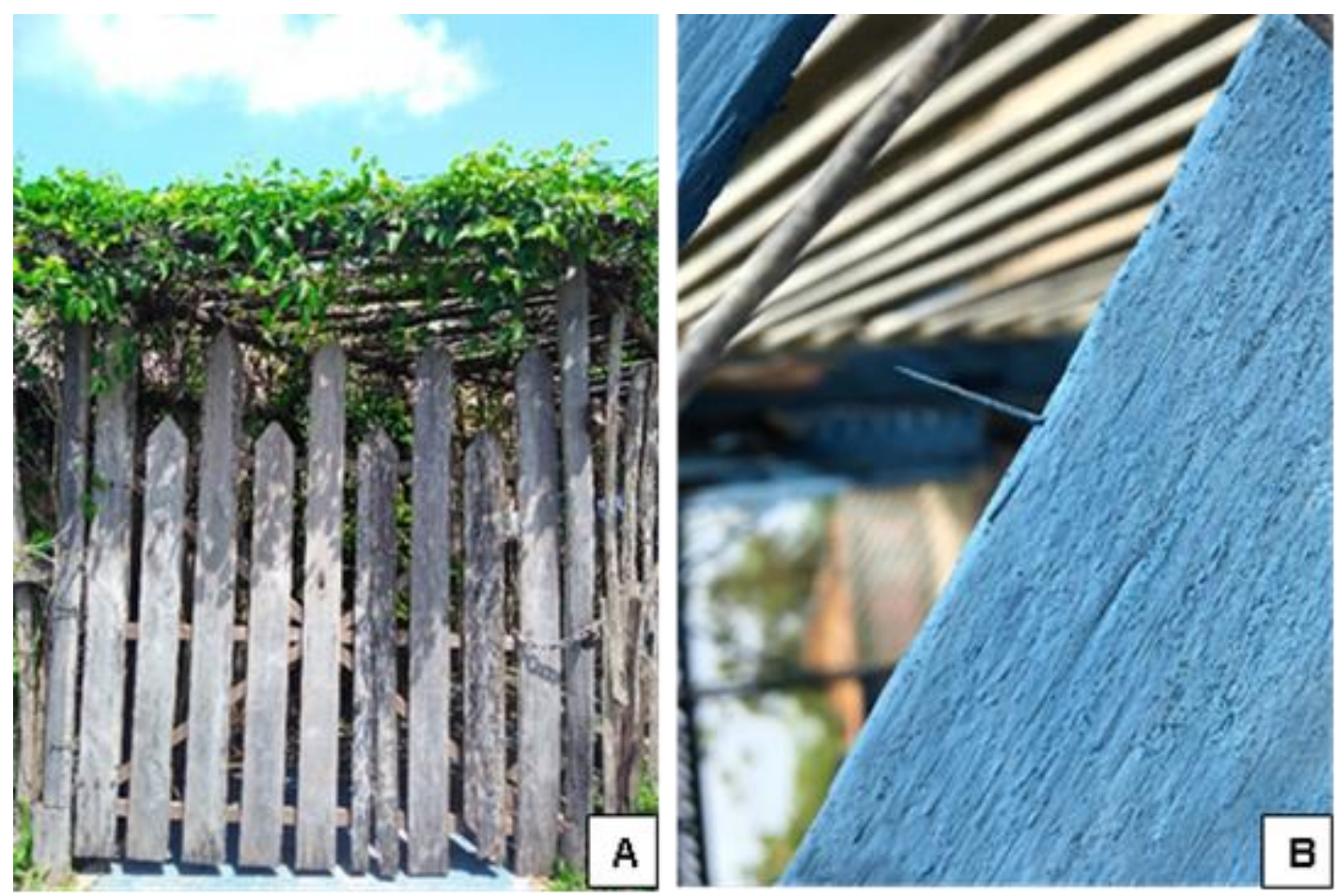

Figura 1. Portões de madeira utilizados durante a coleta de farpas, localizados no município de Soure, Pará. A - Um dos portões utilizados na zona urbana para se obter amostras. B - Farpa encontrada em um portão de madeira da zona rural.

Para a coleta das farpas foi utilizado: pinça, para a retirada da mesma; papel alumínio, para embalá-la; e sacos plásticos para o armazenamento das farpas até que fossem levadas ao Laboratório de Microbiologia e Parasitologia, da Universidade Federal do Pará (UFPA), campus do Marajó - Soure. 
No laboratório, as farpas foram semeadas em meio de cultura Batata Dextrose Ágar (BDA), utilizando-se $39 \mathrm{~g}$ de BDA para um litro de água, e esterilizado em autoclave durante 16 minutos a

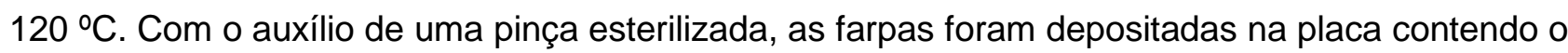
meio de cultura e em seguida, incubadas a temperatura ambiente por um período de 5 a 7 dias. $\mathrm{O}$ processo de semeadura foi realizado dentro da Cabine de Segurança Biológica.

Após o período de incubação, o crescimento fúngico estava visível e, com auxílio de fita adesiva e do corante Lactofenol Azul de Algodão, as lâminas foram preparadas e posteriormente, analisadas em microscopia de luz.

A identificação de cada gênero foi realizada por meio da visualização das estruturas de frutificação características de cada gênero, e com o auxílio de chave de identificação através do "Descriptions of Medical Fungi" (Kidd et al. 2016).

\section{RESULTADOS}

A análise microscópica constatou que houve o crescimento de 137 colônias de fungos filamentosos, sendo 55 dessas pertencentes a fungos demáceos dos gêneros: Cladosporium sp., Curvularia sp., Exophiala sp., Fonsecaea sp. e Phialophora sp. (Figura 2). O que corresponde a 46 de 111 amostras de farpas analisadas. As outras 82 colônias pertenciam aos gêneros: Aspergillus sp., Fusarium sp., Mucor sp., Rhizopus sp. e Penicillium sp.

Das 55 colônias de fungos demáceos, 19 são do gênero Phialophora sp., 12 de Exophiala sp., 10 de Curvularia sp., 08 de Cladosporium sp. e 06 de Fonsecaea sp. (Figura 3). 

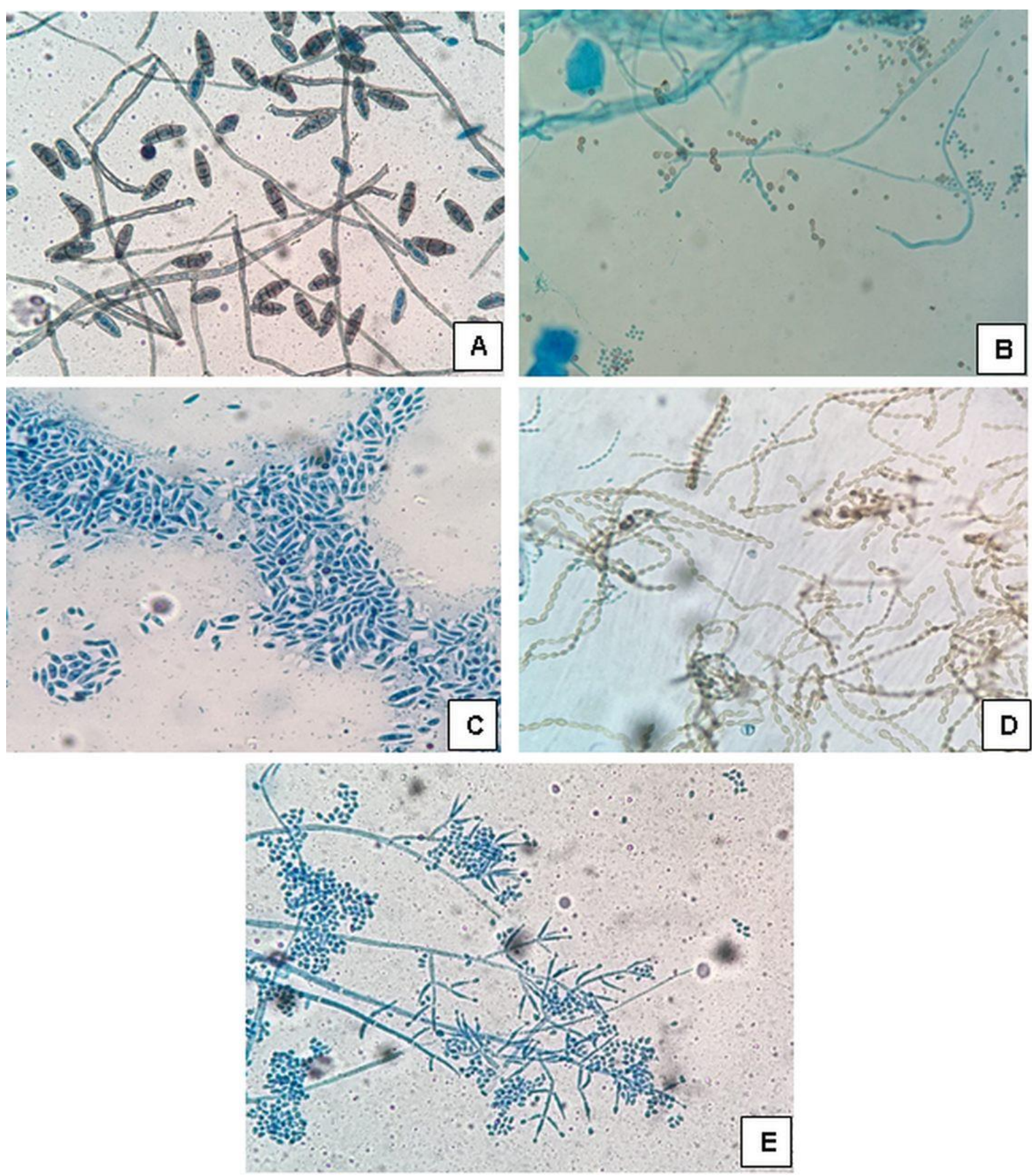

Figura 2. Gêneros de fungos demáceos encontrados em farpas de portões de madeira, no município de Soure, Pará, visualizados em microscopia de luz, aumento de 40x. A. Curvularia sp. B. Fonsecaea sp. C. Exophiala sp. D. Cladosporium sp. E. Phialophora sp. 


\section{FUNGOS DEMÁCEOS}

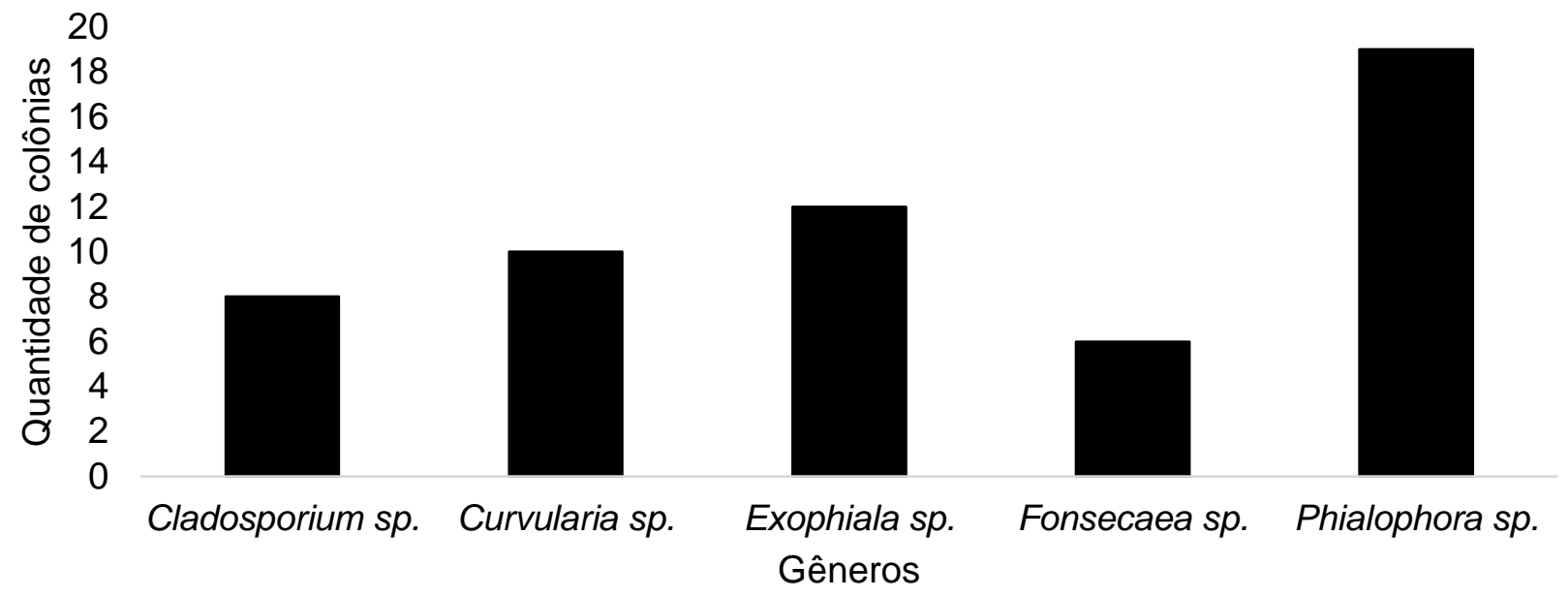

Figura 3. Quantidade total de colônias, por gênero de fungos demáceos, em diferentes anos e estações, no município de Soure, Pará.

No período seco de 2018, houve crescimento de 26 colônias de fungos demáceos, sendo 11 colônias observadas em portões da zona urbana e 15 da zona rural, totalizando cinco gêneros: Cladosporium sp., Curvularia sp., Exophiala sp., Fonsecaea sp. e Phialophora sp. (Figura 4). O gênero mais abundante foi Curvularia sp.

\section{FUNGOS DEMÁCEOS PERÍODO SECO 2018}

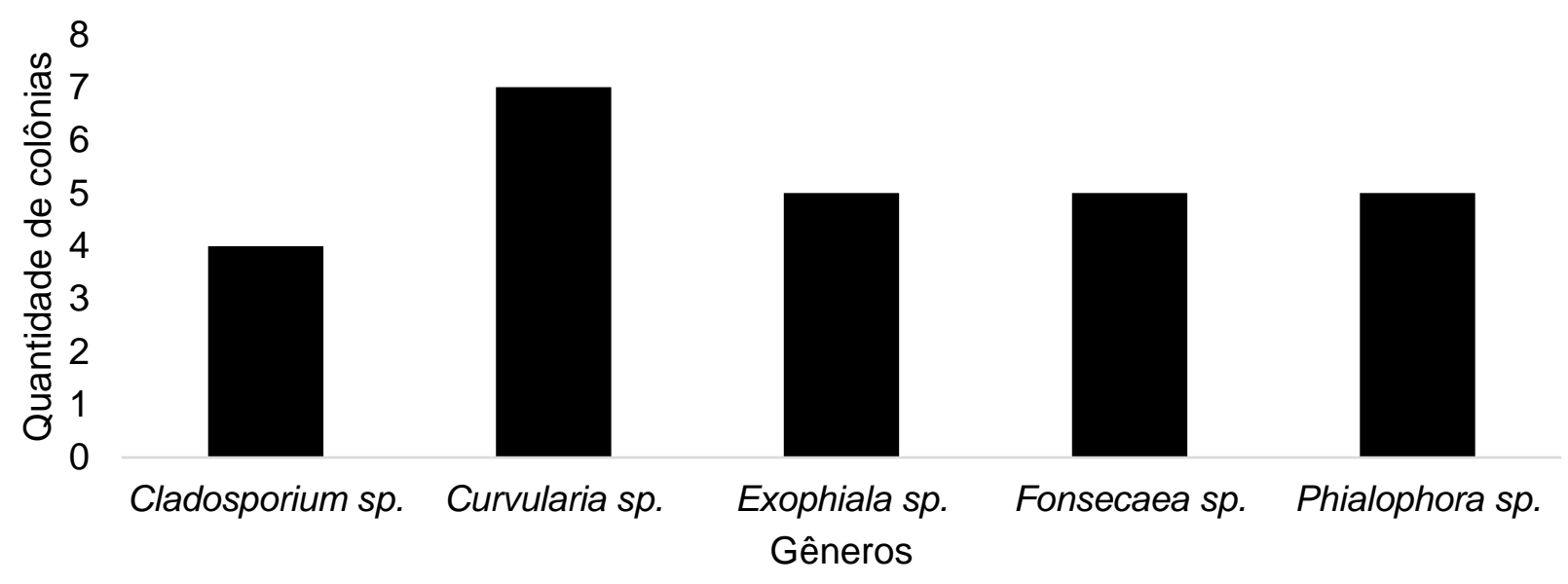

Figura 4. Quantidade total de colônias, por gênero de fungos demáceos, durante o período seco de 2018, no município de Soure, Pará. 
No período chuvoso de 2019, foi registrado o crescimento de 13 colônias, 8 colônias de observadas na zona urbana e 5 na zona rural, dos seguintes gêneros de fungos demáceos: Cladosporium sp., Exophiala sp., Fonsecaea sp. e Phialophora sp. (Figura 5). O gênero mais abundante foi Phialophora sp.

\section{FUNGOS DEMÁCEOS PERÍODO CHUVOSO - 2019}

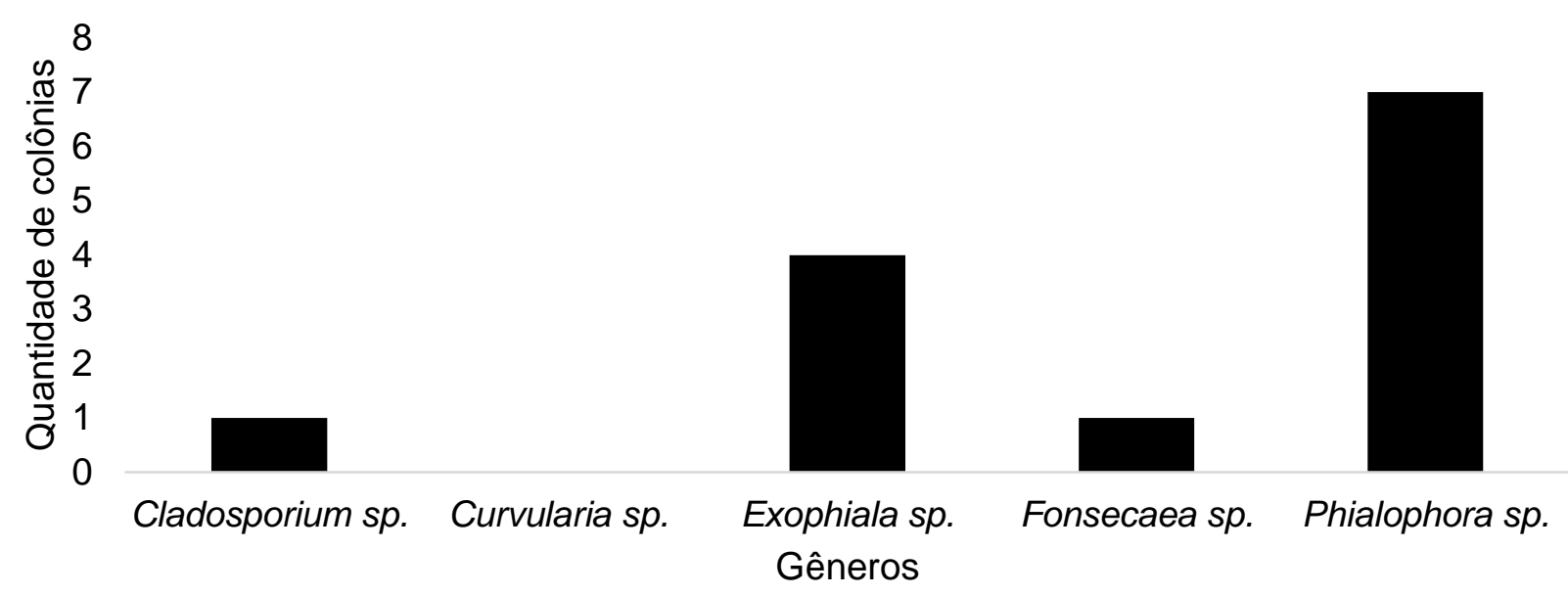

Figura 5. Quantidade total de colônias, por gênero de fungos demáceos, durante o período chuvoso de 2019, no município de Soure, Pará.

No período seco de 2019, ocorreu o crescimento de 16 colônias de fungos demáceos, 4 colônias registradas na área urbana e 12 na área na rural, totalizando quatro gêneros: Cladosporium sp., Curvularia sp., Exophiala sp. e Phialophora sp. (Figura 6). Nesse período, o gênero mais abundante também foi Phialophora sp.

\section{FUNGOS DEMÁCEOS PERÍODO SECO - 2019}

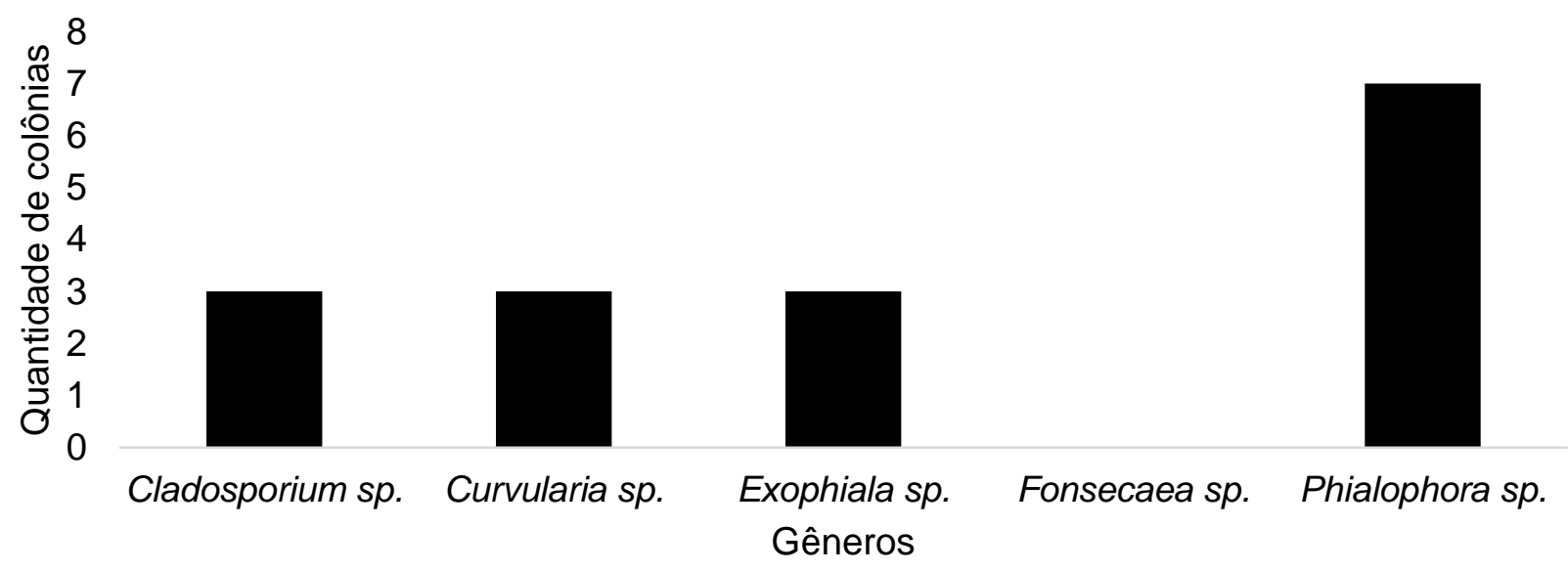

Figura 6. Quantidade total de colônias, por gênero de fungos demáceos, durante o período seco de 2019, no município de Soure, Pará. 
Na zona urbana, houve o crescimento de 23 colônias de fungos demáceos pertencentes aos gêneros: Curvularia sp., Exophiala sp., Fonsecaea sp. e Phialophora sp., sendo este último o mais prevalente e Fonsecaea sp., o menos abundante. Não houve a presença de Cladosporium sp. nesta zona. Na zona rural, houve o crescimento de 32 colônias dos gêneros: Cladosporium sp., Curvularia sp., Exophiala sp., Fonsecaea sp. e Phialophora sp. Novamente, o gênero mais abundante foi Phialophora sp. e o menos frequente, Fonsecaea sp. (Figura 7).

\section{FUNGOS DEMÁCEOS}

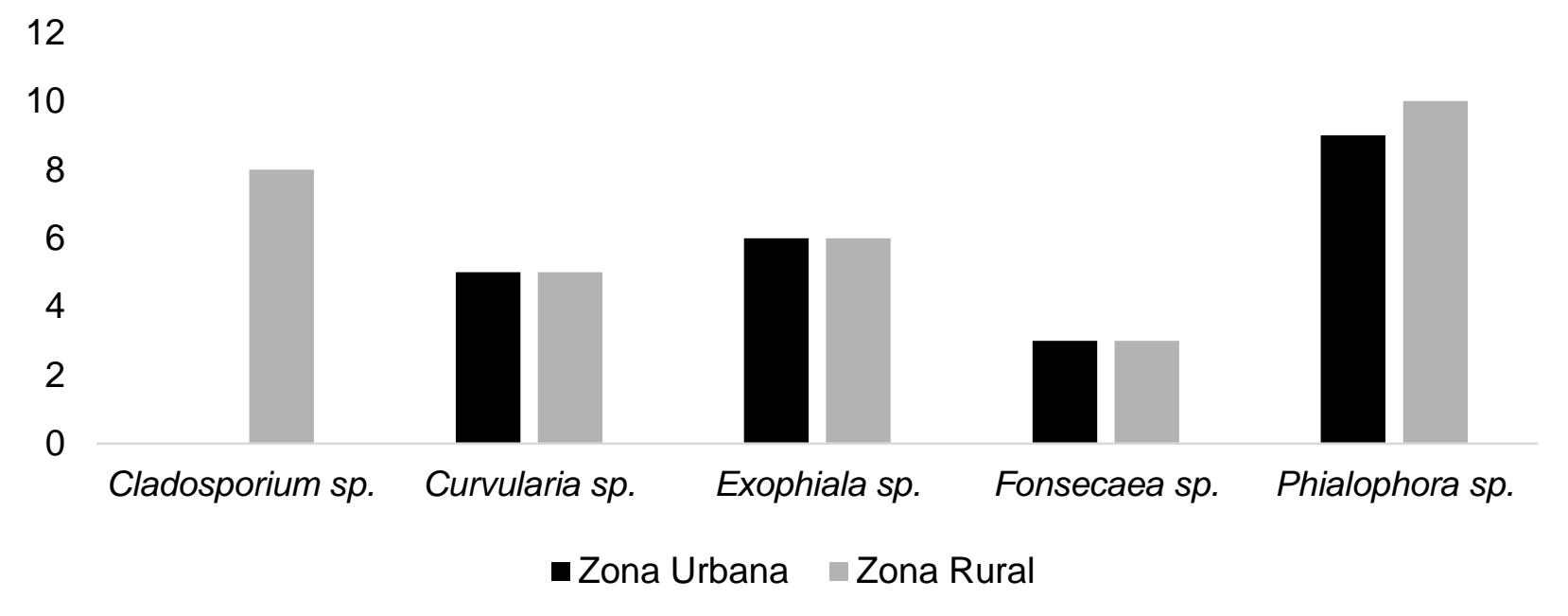

Figura 7. Quantidade total de colônias, por gênero de fungos demáceos, em diferentes anos e estações, correspondente as zonas rural e urbana, do município de Soure, Pará.

\section{DISCUSSÃO}

O gênero mais abundante foi Phialophora, e isso se deve ao fato de que os fungos desse gênero, e fungos demáceos no geral, são saprófitos e muitos portões de madeira estavam em fase de degradação. No estudo de Vicente et al. (2008), esse gênero foi um dos isolados de madeira em estágio de decomposição, no caso, foi a espécie Phialophora verrucosa, que é um dos causadores de Cromoblastomicose. Sabbaga et al. (1994), menciona dois casos de feo-hifomicose causados por espécies do gênero Phialophora no Brasil.

O segundo gênero mais encontrado em portões de madeira foi Exophiala. Esse gênero suporta pequenas e grandes variações de temperatura, uma condição característica do clima da cidade e que pode estar relacionada. Segundo De Hoog et al. (2011), Exophiala, assim como outros gêneros patogênicos a humanos, consegue se desenvolver em temperaturas que variam de 36 a $42{ }^{\circ} \mathrm{C}$. Há espécies patógenas desse gênero como Exophiala dermatitidis, que é capaz de gerar infecção que afeta o cérebro, e a $E$. jeanselmei, que tem sido relacionado a doenças como cromoblastomicose, eumicetoma e feomicose cutânea (Ajantha \& Kulkarni, 2011). No estudo de Alves (2015), este gênero foi isolado de muitas amostras de espinhos. 
O gênero Curvularia foi o terceiro mais frequente. Em um estudo realizado no município, pelo mesmo laboratório desta pesquisa, esse gênero foi encontrado em amostras de espinhos, o que também está relacionado a inoculação traumática (Barbosa e Biancalana, 2016). Segundo Vásquez-del-Mercado et al. (2013), não é comum infecções subcutâneas causadas por este gênero, mas este já foi isolado e descrito em imunossuprimidos e imunocompetentes, inclusive no trabalho citado. Em 1973, foi relatado um caso de micetoma ocasionado por Curvularia lunata no Sudão (Mahgoub, 1973).

Fonsecaea foi o quarto gênero mais observado nos portões de madeira do município. Sua ocorrência pode estar ligada ao fato dele ser um gênero que se desenvolve bem em climas tropicais, como o da Amazônia. Ademais, ele foi encontrado em portões que estavam em fase de degradação, o que pode ter favorecido seu crescimento devido a seu hábito saprófito. Isso corrobora com o estudo de Alves (2015), onde uma espécie do gênero Fonsecaea foi isolada de amostra de madeira em decomposição. Uma das espécies desse gênero, F. pedrosoi, é uma das principais causadoras de cromoblastomicose na Amazônia (Silva et al., 1999; Salgado, 2007).

O gênero Cladosporium foi o que apresentou menos registros em portões de madeiras comparado com os outros. No estudo de Bonfim et al. (2016), os fungos desse gênero foram classificados como generalistas, classificação essa que não se aplica a este estudo, visto que o gênero foi encontrado em menor quantidade e apenas na zona rural. De acordo com Asan et al. (2003), ele é o gênero saprófito mais comum e, frequentemente encontrado onde há plantas herbáceas mortas e outras matérias orgânicas. É um gênero bastante visto como infeccioso (De Hoog et al., 2014).

Dessa forma, os resultados desse estudo advertem a população sobre os riscos que as farpas presentes nos portões de suas casas podem causar a saúde, visto que há organismos patogênicos que as colonizam.

Os cinco gêneros citados acima foram encontrados em farpas de madeira retiradas de áreas dos portões onde a população normalmente manuseia para abri-los, já que esses fragmentos, quando presentes, costumam causar traumas nos indivíduos, como pequenos arranhões. Segundo Vicente et al. (2008), geralmente em humano, a inoculação traumática é a porta de entrada do fungo. O mesmo pode continuar na região do ferimento através do desenvolvimento de hifas, podendo gerar uma lesão no local ou se espalhar para outras regiões corpóreas devido a reprodução por brotamento, causando até micoses mais graves (De Hoog, 1993).

Em relação a quantidade de colônias de fungos demáceos encontradas, houve diferença entre as zonas urbana e rural. Isso pode estar relacionado ao fato de que na zona urbana, o fluxo de pessoas é mais intenso, o que afeta o carreamento dos conídios, diferentemente da zona rural, onde o fluxo é menor. Segundo Lacaz et al. (2002), essa diferença pode estar ligada tanto ao fluxo de indivíduos transitando a zona quanto a fatores ambientais como temperatura, ventilação e umidade. 
Também houve diferenças entre as estações seca e chuvosa da nossa região, que é um clima característico da Amazônia, onde as estações secas apresentaram maior número de colônias. Possivelmente, isso ocorreu em virtude de esses fungos serem capazes de suportar altas temperaturas e ambientes secos. Segundo Gostincar et al. (2010), do ponto de vista ecológico, esses fungos tem a capacidade de suportar baixos valores de $\mathrm{pH}$ e altas temperaturas. Selbmann et al. (2005), relatam que além de tolerar grandes concentrações salinas e radiação UV, esses organismos conseguem suportar ambientes secos.

A presença dos fungos demáceos em farpas de portões de madeira necessita de uma atenção singular, visto que a população os manuseia diariamente e todos os gêneros encontrados, mesmo que não identificados a nível de espécie, apresentam indivíduos patogênicos que podem causar micoses subcutâneas severas, como cromoblastomicose, micetoma e feo-hifomicose, através da inoculação de farpas contaminadas por eles.

\section{CONCLUSÃO}

Phialophora foi o gênero de fungos demáceos mais frequente, ocorrendo em ambas as estações seca e chuvosa de 2019, nas zonas rural e urbana do município. Em relação ao período seco de 2018, o gênero mais encontrado em farpas de portões de madeira foi Curvularia.

Nas estações secas houve maior ocorrência de fungos demáceos quando comparadas com a estação chuvosa.

\section{AGRADECIMENTOS}

Agradecemos a UFPA, ao Laboratório de Microbiologia e Parasitologia e ao CNPq, por apoiar essa pesquisa.

\section{REFERÊNCIAS}

Afsarian, M. H.; Tahereh, S.; Mahdi, A.; Mehdi, T. S.; Hamid, B. Phaeohypomycosis due to dematiaceous fungi: a Review of the literatures. Journal Mazand University of Medical Science, 22(92), 109-135, 2012.

Ajantha, G. S.; Kulkarni, R. D. Cladophialophora bantiana, the neurotropic fungus - a Mini Review. Journal of Clinical and Diagnostic Research, 5(6), 1301-1306, 2011.

Alves, M. J. Estudo de fungos melanizados em amostras ambientais de áreas rurais do Amazonas e avaliação da sua relação com os fungos melanizados causadores de micoses. $104 \mathrm{f}$. Dissertação (Mestrado em Saúde, Sociedade e Endêmias na Amazônia) - Universidade Federal do Amazonas, Manaus. 2015.

Asan, A.; Kirgiz, T.; Sen, B.; Camur-Elipek, B.; Guner, U.; Guher, H. Isolation, identification and seasonal distrubution of airborne and waterborne fungi in Terkos Lake (Istanbul-Turkey). Journal of Basic Microbiology, 43(2), 83-95, 2003.

Badali, H.; Gueidan, C.; Najafzadeh, M. J.; Bonifaz, A.; van den Ende, A. H. G. G.; De Hoog, G. S. Biodiversity of the genus Cladophialophora. Studies in Mycology, 61, 175-191, 2008. 
Barbosa, G. S.; Biancalana, F. S. C. Avaliação da ocorrência de fungos demáceos em áreas de manguezal da Reserva Extrativista Marinha de Soure-Pa. In: VIII CONGRESSO BRASILEIRO DE MICOLOGIA, 2016. Anais do VIII Congresso Brasileiro de Micologia, 2016. Florianópolis: Universidade Federal de Santa Catarina, 2016.

Bonfim, J. A.; Vasconcellos, R. L. F.; Baldesin, L. F.; Sieber, T. N.; Cardoso, E. J. B. N. Dark septate endophytic fungi of native plants along an altitudinal gradient in the Brazilian Atlantic forest. Fungal Ecology, 20, 202-210, 2016.

Castro, L. G. M. Chromomycosis: A therapeutic challenge. Clinical Infectious Diseases, 15(3), 553, 1992.

Cucé, L. C.; Salebian, A.; Gatti C. F.; Sampaio, S. A. P. Cromomicosis: Estudio de 37 casos. Revista Argentina de Dermatologia, 64,1-1, 1983.

De Hoog, G. S. Evolution of black yeasts: possible adaptation to the human host. Antonie Van Leeuwenhoek. 63, 105-109, 1993.

De Hoog, G. S.; Guarro, J.; Gené, J.; Figueras, M. J. Atlas of clinical fungi: the ultimate bench tool for diagnostic. 4. ed. Washington: Editora Amer Society for Microbiology, 2014.

De Hoog, G. S.; Vicente, V. A.; Najafzadeh, M. J.; Harrak, M. J.; Badali, H.; Seyedmousavi, S. Waterborne Exophiala species causing disease in cold blooded Animals. Persoonia, 27, 46-72, 2011.

Fan, Y. M.; Huang, W. M.; Li, S. F.; Wu, G. F.; Li, W.; Chen, R. Y. Cutaneous phaeohyphomycosis of foot caused by Curvularia clavata. Mycoses, 52, 544-546, 2008.

Fothergill, A. W. Identification of dematiaceous fungi and their role in human disease. Clinical Infectious Diseases, 22,179-184, 1996.

Gold, W. L.; Vellend, H.; Salit, I. E.; Campbell, I.; Summerbell, R.; Rinaldi, M.; Simor, A. E. Successful treatment of systemic and local infections due to Exophiala species. Clinical Infectious Diseases, 19(2), 339-341, 1994.

Gostinčar, C.; Grube, M.; De Hoog, G. S.; Zalar, P.; Gundecimerman, N. Extremotolerance in fungi: evolution on the edge. Federation of European Microbiological Societies (FEMS), 71(1), 2-11, 2010.

Hoffmann, C. C.; Danucalov, I. P.; Purim, K. S. M.; Queiroz-Telles, F. Infecções causadas por fungos demáceos e suas correlações anátomo-clinicas. Anais Brasileiros de Dermatologia, 86(1), 138-41, 2011.

Jacobson, E. S. Pathogenic roles for fungal melanins. Clinical Microbiology Reviews, 13(14), 708$717,2000$.

Kidd, S.; Halliday, C.; Alexiou. H.; Ellis, D. Descriptions of medical fungi. 3. ed. Adelaide: Newstyle Printing, 2016.

Lacaz, C. S.; Porto, E.; Martins, J. E. C.; Heins-Vaccau, E. M.; Melo, N. T. Tratado de Micologia Médica. 9. ed. São Paulo: Sarvier, 2002.

Lopes, L. F. D. Amostragem. In: Lopes, L. F. D. Apostila de Estatística. Santa Maria: Editora UFSM, 2003, p.85-94.

Mahgoub, E. S. Mycetomas caused by Curvularia lunata, Madurella grisea, Aspergillus nidulans, and Nocardia brasiliensis in Sudan. Sabouraudia, 11(2), 179-82, 1973. 
Najafzadeh, M. J.; Gueidan C.; Badali, H.; Gerrits Van Den Ende, A. H. G.; Xi, L.; De Hoog, G. S. Genetic diversity and species delimitation in the opportunistic genus Fonsecaea. Medical Mycology, 47, 17-25, 2011.

Nimer, E. Climatologia do Brasil. 2. ed. Rio de Janeiro: IBGE, Departamento de Recursos Naturais e Estudos Ambientais, 1989.

Queiroz-Telles, F.; Nucci, A.; Colombo, A. L.; Tobón, A.; Restrepo, A. Mycoses of implantation in Latin America: an overview of epidemiology, clinical manifestations, diagnosis and treatment. Medical Mycology, 49(3), 225 - 236, 2011.

Reis, N. R. Morcegos da região de Manaus e suas relações com fungos patogênicos. Semina, 12(3), 255-262, 1982.

Revankar, S. G. Phaeohyphomycosis. Infectious Disease Clinics of North America, 20(6), 609-620, 2006.

Revankar, S. G.; Sutton, D. A. Melanized fungi in human disease. Clinical Microbiology Reviews, 23(4), 884-928, 2010.

Rubin, H. A.; Bruce, S.; Rosen, S.; Mcbride, M. E. Evidence for percutaneous inoculation as the mode of transmission for chromoblastomycosis. Journal of American Academy of Dermatology 25(5), 951-954, 1992.

Sabbaga, E.; Tedesco-Marchesi, L.; Lacaz, C.; Cucé, L.; Salebian, A.; Heins-Vaccari, E.; Sotto, M.; Valente, N.; Porto, E.; Levy Neto, M. Feo-hiomicose subcutânea causada por Exophiala jeanselmei: relato de três casos em pacientes transplantados renais. Revista do Instituto de Medicina Tropical de São Paulo, 36(2), 175-183, 1994.

Salgado, C. G. Micoses Profundas. In: Simpósio Patologia TropicaL, 2007. Anais da 59a Reunião Anual da SBPC, 2007. Belém: Universidade Federal do Pará, 2007.

Santos, M. A Urbanização Brasileira. 1. ed. São Paulo: Hucitec, 1993.

Selbmann, L.; De Hoog, G. S.; Mazzaglia, A.; Friedmann, E. I.; Onofri, S. Fungi at the edge of life: cryptoendolithic black fungi from Antarctic desert. Studies in Mycology, 51, 1-32, 2005.

Shelton, B. G.; Kirkland, K. H.; Flanders, W. D.; Morris, G. K. Profiles of airborne fungi in buildings and outdoor environments in the United States. Applied and Environmental Microbiology, 68(4), 1743-1753, 2002.

Silva, J. P.; Souza, W.; Rozental, S. Chromoblastomycosis: a retrospective study of 325 cases on Amazonic region. Mycopathologia, 143(3), 171-175, 1999.

Vásquez-Del-Mercado, E.; Lammoglia, L.; Arenas, R. Subcutaneous phaeohyphomycosis due to Curvularia lunata in a renal transplant patient. Revista Iberoamericana de Micologia, 30(2), 116-118, 2013.

Vicente, V. A.; Attili-Angelis, D.; Pie, M. R.; Queiroz-Telles, F.; Cruz, L. M.; Najafzadeh, M. J.; De Hoog, G. S.; Zhao, J.; Pizzirani-Kleiner, A. Environmental isolation of black yeast-like fungi involved in human infection. Studies in Mycology, 61, 137-144, 2008.

Vicente, V. A.; Attili-Angelis, D.; Queiroz-Telles, F.; Pizzirani-Kleiner, A. Isolation of Herpotrichellacious fungi from the environment. Brazilian Journal of Microbiology, 32, 47-51, 2001.

Wong, E. H.; Revankar, S. G. Dematiaceous molds. Infectious Disease Clinics of North America, 30(1), 165-78, 2016. 
Zhao, J.; Zeng, J.; De Hoog, G. S.; Attili-Angelis, D.; Prenafetaboldú, F. X. Isolation and identification of black yeasts by enrichment on atmospheres of monoaromatic hydrocarbons. Microbial Ecology, 60(1),149-156, 2010.

Zolnerkevic, I. O lado negro das leveduras. Revista Unesp Ciência, 21, 26-28, 2011. 